\title{
The Existence
}

\section{Martin Vlcek}

Functionality, Non-Profit Research Group, Prague, Czech Republic

*Corresponding Author:Martin Vlcek, Functionality, Non-Profit Research Group, Prague, Czech Republic, Tel: 727164850, Email: martin.vlcek@efunctionality.eu

Received Date: February 11, 2020; Accepted Date: February 17, 2020; Published Date; February 20, 2020.

Citation: Martin Vlcek. (2020). The Existence, J. Clinical Research Notes. 1(1). Doi: 10.31579/crn.2020/007

Copyright: (C) 2020. Martin Vlcek, This is an open-access article distributed under the terms of the Creative Commons Attribution License, which permits unrestricted use, distribution, and reproduction in any medium, provided the original author and source are credited.

\section{Introduction}

Living objects can be understood as systems that are able to evaluate their own existence and, based on experience, to respond optimally to its development.

Existence in living systems materializes in a part that has a fundamentally different architecture than the rest of the system, and will continue to be called Dark Matter - DM.

For the object is essential interconnection with other DM. Its existence in DM must be linked to other existence, to DM as a whole and that is the structure of DM. The DM object itself has no structure and that is its essence. The structure is in its interconnection with the surrounding DM. Around DM there are evaluating and influencing parts that form its boundary with the rest of the living system - NM.

Global DM thus realizes the "philosophical" unity of the functioning of systems, the material unity of the "universe."This work is based primarily on the results of model calculations above the US stock markets. The red thread is a presumption of the general validity of the properties of living systems, and the results are thus illustrated on the human organism and the society.

\section{The Existence}

The level of existence of a living object is a function of the satisfaction of its elements, manifested as a tendency to leave the object. But satisfaction is an internal state, unlike, for example, the success of an element in the system. Success is a valuation unit in NM and, for example, in society is expressed in money.

The involvement of DM in the existence of an object is "simple". Under certain circumstances, DM creates an output that enters and activates the NM. The NM then performs all system steps as it corresponds to its structure, and only at this point in time can it be said that it exists. DM is a kind of pace-maker. At the same time, the system time is also defined. Beyond this activity the object exists only through its elements. However, they have their own DM, and this is an analogous situation.

DM produces output in the following circumstances. Generally, it is a situation which is a manifestation of an "endangered" existence and this is a manifestation of an event in the satisfaction of the elements. Everything is conducted by the border regions around DM, only they are able to capture its size - the measure of existence.

\section{Inputs into DM}

Based on model calculations of the regularities in the development of stocks it seems that three sources are used as a source for quantifying the existence and identifying and responding adequately to the event. On the one hand, it is information about the current development of the measure of existence. For the subsequent optimal response, the object needs information about the current satisfaction of the elements as well as the whole object.

Satisfaction of the elements, which is not observable in itself, is evaluated from their success - at least in economic situations. Success balance is used in certain areas. From a system point of view, a given area - a group of, for example, similarly specialized or situated elements - is relatively "satisfied" if its elements are relatively equally successful. It seems to be a kind of "envy" criterion, but it is an important source of information about element satisfaction.

The second source is information about the satisfaction of the object as a whole. It is analogous to the satisfaction of the element - ie it is a monitoring of the migration tendency. In this case, the cross-border movement of the "goods and services" of the object - its relations with its surroundings - is monitored. This movement is apparently interpreted as a certain precursor to the migration pressure of the elements. Among other things, the imbalances of these relationships formally represent the movement of an object in a system of analogous objects. It is a manifestation of the degree of dissatisfaction of the object as a whole and its possible "migration".

\section{Development of DM}

The object evaluates the existence on the border with DM and monitors the sequence of its changes with its behavior. Because it seeks to maximize its existence, it learns on history how to proceed in certain situations - sequences of measure of existence over time - to optimize. Thus, if it finds a known event in the development of DM, the consequence of which has been positive for existence, it reactivates the system as a whole.

\subsubsection{Firms}

In this case, the boundary between DM and NM are real-estate investment trusts - reit. These are companies doing business in real estate, especially in investing in them. The overall rate of this investment and its dynamics seems to best reflect the characteristics of DM.

\subsubsection{Human organism}

The location of the DM boundary in the nervous system of humans and higher animals in general is not very clear. It is generally in the part of the nervous system that surrounds cerebrospinal fluid. The placement of the output part, the area from which the system activating DM output is entering the brain, seems clearer. This is probably located in the stem. In 
contrast, the entry into DM is in the cerebral cortex. In the spinal cord, information about the boundary and the state of satisfaction of the whole object seems to prevail.

Evaluation in the entry area to DM is probably performed by the highest neural layer. The output from it to DM is activity, transmitted e.g. by the dynamics of hydrogen bridges and free hydrogens themselves, which spreads much faster than the action potential of neurons. As a result, changes are relatively immediate and occur throughout DM. And this is the essence of DM functioning - as a whole and at a different time than NM.

However, the result of entering DM is a state variable that could seem to be illogically given by the state of "dynamics" of hydrogen bridges.

\subsubsection{Society}

In human society, DM is in the state budget. Individual departmental ministries assess the needs of their department, or experience with different cash flows, and create requirements accordingly. In summary, there is a requirement for the state budget, which is finally approved, ie created and subsequently spent. In the case of the state, the existence of an object is the whole time of spending means as many elementary existence is approved as high is the budget.

\section{Conclusion}

The materialization of the concept of existence is certainly not only of formal significance. E.g. in an object called "family" (-) its interpretation can have a positive effect on the stability of the family - partnership. Strengthening the common budget, spending it to deal with problematic, partitioning situations, according to an analogy approach, should not remain without impact on existence.

\section{Reference}

1. Martin Vlček: Dark Matter in our Ordinary Life, (2018); Amazon in Wroclaw; ISBN 9781718882836

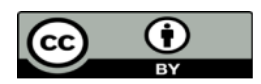

This work is licensed under Creative Commons Attribution 4.0 License
Ready to submit your research? Choose Auctores and benefit from:

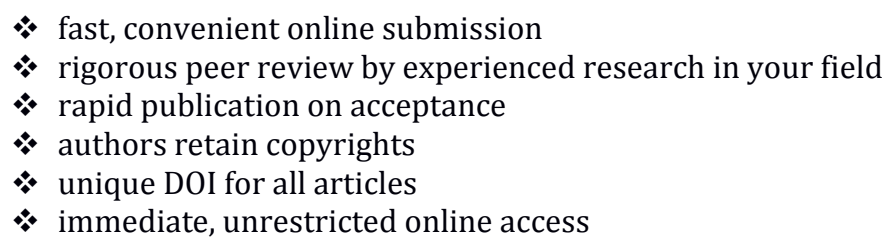

At Auctores, research is always in progress.

Learn more www.auctoresonline.org/journals/clinical-research-notes-

DOI: $10.31579 /$ crn.2020/007 